\title{
Genetic Influences on Spatial Ability: Transmission in an Extended Kindred
}

\author{
Susan L. Smalley, ${ }^{1,5}$ Andrea Lee Thompson, ${ }^{2}$ M. Anne Spence, ${ }^{3}$ \\ W. John Judd, ${ }^{4}$ and Robert S. Sparkes ${ }^{3}$
}

Received 4 Dec. 1986-Final 9 Feb. 1988

Transmission of six spatial tests, Card Rotations, Cube Comparisons, Group Embedded Figures, Hidden Patterns, Mental Rotations, and portable Rod and Frame, is examined among 73 members in four generations of an extended kindred. Nonadditive genetic variance is substantial for one of the six tests, Card Rotations. Whether this nonadditive genetic variance is due to a major autosomal gene is equivocal based on results from segregation and linkage analysis. There is no evidence for genetic variance for Mental Rotations or Hidden Patterns, in contrast to previous findings suggesting major gene involvement (Ashton et al., 1979). If spatial ability is due, in part, to an autosomal major gene, the gene has variable expression (reflected in different tests) or genetic heterogeneity is pronounced.

KEY WORDS: segregation analysis; linkage; heritability; spatial ability.

\section{INTRODUCTION}

Spatial ability has been the focus of numerous behavior genetic studies of cognition. Despite intense investigation, debate continues as to whether

This work was supported by National Institutes of Health Grant HD-05615.

${ }^{1}$ Department of Psychiatry, University of California School of Medicine, Los Angeles, California 90024.

${ }^{2}$ Eric Marder and Associates, 122 East 42nd Street, New York, New York 10168.

${ }^{3}$ Division of Medical Genetics, Departments of Psychiatry, Medicine and Pediatrics, University of California School of Medicine, Los Angeles, California 90024.

${ }^{4}$ Department of Pathology, University of Michigan, Ann Arbor, Michigan 48109.

${ }^{5}$ To whom correspondence should be addressed at 48-241 NPI, Department of Psychiatry, UCLA School of Medicine, 760 Westwood Plaza, Los Angeles, California 90024. 
a single major gene is involved in transmission of certain spatial skills. O'Connor (1943) first proposed X-linked recessive inheritance for spatial ability, with high ability recessive to low ability. Early studies (Corah, 1965 ) tested the X-linked model by comparing patterns of familial correlations (mother-son, mother-daughter, father-son, father-daughter) with those expected under the model. While patterns were consistent with $\mathrm{X}$-linked inheritance in a few early studies, (for review, see Vandenberg, 1979), sample sizes were small and later investigations using large samples failed to replicate these findings (Loehlin et al., 1978; DeFries et al., 1979). Jardine and Martin (1984) used pedigree analysis in twin families to test for $\mathrm{X}$-linked genes in spatial ability and found no evidence supporting Xlinked inheritance.

Given a lack of support for $\mathrm{X}$ linkage, polygenic or multifactorial inheritance is often invoked to explain genetic variation in spatial ability. However, Aston and colleagues (1979) found segregation patterns among 1260 families in the Hawaii Family Study of Cognition consistent with a major autosomal gene for three of six spatial tests. Although the spatial scores were adjusted for significant skewness, kurtosis was not adjusted and the extent to which deviations from normality in this moment influenced their findings was unclear. Simulation studies have shown that deviations from normality of third and fourth moments about the mean can lead to false detection of a major gene (Eaves, 1983).

In the present investigation we examine genetic influences on spatial ability, adjusted for significant skewness and kurtosis, in four generations of an extended kindred. The rationale for using this sampling design is twofold. First, genetic heterogeneity is reduced in a single family compared to large numbers of nuclear families. Identification of a major gene, if present, will be less confounded by alternative etiologies (e.g., phenocopies, other modes of transmission). Second, multigeneration kindreds are more informative than two generation families in linkage investigations (Elston and Bonney, 1984).

\section{MATERIALS AND METHODS}

\section{Subjects}

The sample consists of 73 members ( 32 males and 41 females) in four generations stemming from one pair of original parents. The kindred was originally ascertained by two of the authors (A.T. and M.A.S.) because of its size and availability for study as part of an ongoing investigation of marker by marker linkage in humans. The kindred is European derived and split geographically into two regions of the United States. The larger fraction resides in a rural community in Ohio and the smaller fraction 
resides in a large urban setting in California. The family structure of the 73 subjects, with additional connecting family members, is shown in Fig.

1. As indicated in the figure, virtually all subjects are from generations II, III, and IV, with numbers totaling 20,29 , and 22 , respectively. There is one pair of monozygotic female twins in generation IV. Subjects measured on at least one cognitive test are denoted by a shaded symbol in Fig. 1.

\section{Measures}

Subjects 12 years of age and older were administered a battery of cognitive tests measuring three areas of cognitive functioning, spatial ability, perceptual speed, and memory. The 12 paper-and-pencil cognitive tests were administered in two 1-h sessions (separated by a short break) to groups of 10-15 individuals. Two of these tests (GEF and HP) are measures of field dependence/independence. Each requires the subject to identify a simple pattern among complex designs. Three tests, CC, CR, and MR, are measures of spatial orientation, the ability to "perceive spatial patterns or to maintain orientation with respect to objects in space" (Ekstrom et al., 1976, p. 149). Each requires the subject to rotate an object mentally (a geometric figure- $\mathrm{CR}$, a cube-CC, or a complex blockMR) and identify it among several alternative figures. The tests and order of administration are shown in Table I. Testing was done by two of the authors (A.T. and S.S.) and a clinical psychologist trained in test administration, Dr. Vivian Clemins.

All subjects 8 years of age and older were individually measured on the portable Rod and Frame (Oltman, 1968), a measure of field dependence/independence. The task consists of a series of trials where subjects adjust a tilted rod to a vertical position within a distracting tilted frame. A subject is considered more field dependent the larger the score.

Subjects were measured on 24 blood markers, red-cell antigens, and serum enzymes $[A B O, A C P 1, A D A, A K 1, A M Y 2, B F, C H E 2, E S D, F Y$, GALT, GC, GPT, HP, IGHG(GM),JK, KEL, LE, MNS, P1, PGD, $P G M 1, P G P, R H$, and $X G$ ]. Autosomal blood markers were typed by R.S.S., and W.J.J. performed $X G$ typing. Subjects were also measured on PTC tasting ability (Harris and Kalmus, 1949) and dermatoglyphics (Holt, 1968).

\section{Methods}

Analysis of the spatial data consists of five stages. First, raw scores are age and sex adjusted using the following procedure. Scores are 

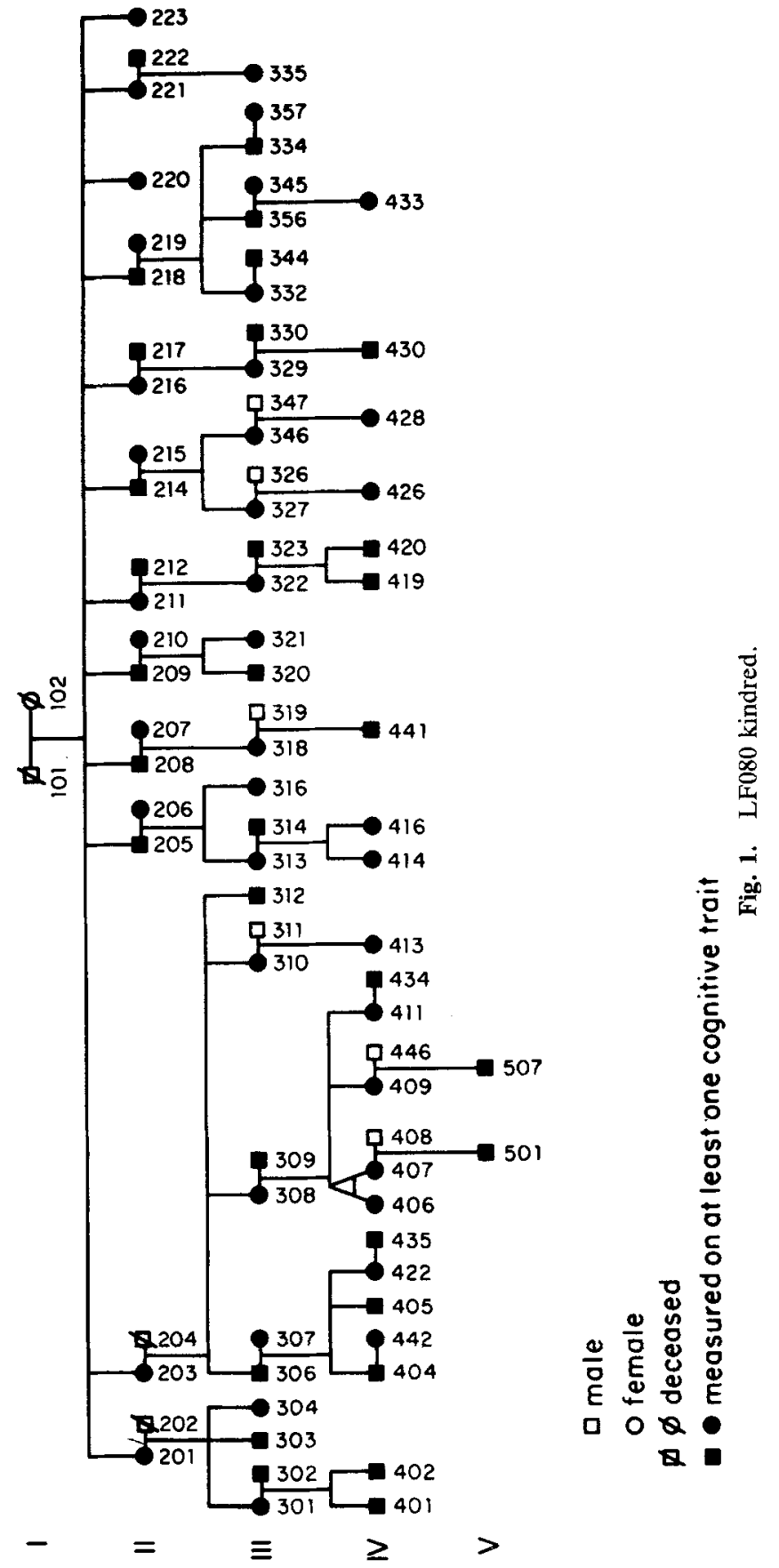
Table I. Cognitive Paper-and-Pencil Tests and Order of Administration

\begin{tabular}{lc}
\hline \multicolumn{1}{c}{ Test (abbreviation) } & Order of presentation \\
\hline First hour of testing & 6 \\
Auditory Number Span (ANS) & \\
Card Rotations (CR) $^{a, b}$ & 1 \\
Cube Comparisons (CC) $^{a, b}$ & 4 \\
Finding A's (FAS) $^{a}$ & 5 \\
Hidden Patterns (HP) $^{a, b}$ & 7 \\
Number Comparisons (NC) $^{a}$ & 3 \\
Visual Memory Immediate (VMI) $^{c}$ & 2 \\
Visual Memory Delayed (VMD) $^{c}$ & 8 \\
Second hour of testing & 1 \\
Group Embedded Figures (GEF) $^{d}$ & 4 \\
Identical Pictures (IP) $^{a}$ & 2 \\
Mental Rotations (MR) & 3 \\
Picture Number (PN) & \\
\hline
\end{tabular}

${ }^{a}$ Source: Education Testing Service, (Ekstrom et al., 1976).

${ }^{b}$ Spatial test.

${ }^{c}$ Source: Wilson and Vandenberg (1977).

${ }^{d}$ Source: Shepard and Metzler (1971); Vandenberg and Kuse (1978).

"smoothed" using a median smoothing technique to minimize the effects of outliers on defining age by trait relationships (Tukey, 1977). The process consists of taking running medians of a variable, $Y$ (spatial test), ordered by values in a variable, $X$ (age). An age function within sex groupings for the smoothed scores is defined using a polynomial regression (BMDP5R) or by fitting two linear regressions defined in two age groupings (BMDP1R). These age functions are then used to define residual scores from the original (raw) scores. These age- and sex-adjusted residual scores are used in subsequent analyses.

Second, age- and sex-adjusted scores are transformed if skewness or kurtosis is significantly different from that expected under a normal distribution. Since skewness and/or kurtosis can lead to false detection of a major gene (Eaves, 1983), we transformed age- and sex-adjusted scores to reduce both third and fourth moments if either differed significantly from that expected under a normal distribution. We fit a series of power and logarithmic transformations and chose the transformation which resulted in skewness closest to zero while keeping kurtosis close to its expected value. All scores are standardized to a mean of zero and variance of one for analyses.

Third, a components-of-variance model for pedigree data (Lange $e t$ al., 1976) is used to test the null hypothesis of "no genetic variation" and to estimate genetic and environmental variance comonents. Under the 
model, variation in spatial test performance is due to additive gene effects, $V_{\mathrm{A}}$, nonadditive genetic variance, $V_{\mathrm{D}}$, and nonfamilial environmental variance, $V_{\mathrm{E}}$. We use the notation $V_{\mathrm{A}}$ and $V_{\mathrm{D}}$ to represent familial variance. $V_{\mathrm{A}}$ reflects additive genetic and/or shared environment of relatives other than siblings, while $V_{\mathrm{D}}$ reflects nonadditive genetic and shared sibling environmental variance. $V_{\mathrm{E}}$ reflects nonfamilial variance. A test of the null hypothesis is done by comparing the likelihood of the data under the full model (where $V_{\mathrm{A}}, V_{\mathrm{D}}$, and $V_{\mathrm{E}}$ are present) with the likelihood under the restricted model $\left(V_{\mathrm{A}}=V_{\mathrm{D}}=0\right)$. Whether such genetic variance is due to a major gene or polygenes is tested in the fourth stage of analysis.

The presence of a major gene is tested by comparing the fit of the kindred spatial score data under a mixed genetic model (major gene and polygenes present) with a restricted model of polygenes alone. A model which includes both major gene and polygenes was developed by Morton and MacLean (1974). Under the model, a trait " $X$ " results from the joint, additive, unobservable contributions of a major transmissible effect $(\mathrm{g})$, a multifactorial component $(c)$, and a random nontransmissible component $(e)$. The major effect results from segregation at a single autosomal locus with two alleles, $A$, and $a$, and genotypes, $A A, A a$, and $a a$.

Segregation at the major locus is specified by the gene frequencies, genotype frequencies, and transmission probabilities (Elston and Stewart, 1971). Transmission probabilities, $T_{1}, T_{2}$, and $T_{3}$, represent the probabilities of transmitting allele $A$ for genotypes $A A, A a$, and $a a$, respectively. Under an autosomal mode of transmission, $T_{1}=1.0, T_{2}=.5$, and $T_{3}=$ 0 . Multifactorial transmission can be specified through the parent-offspring correlation conditional on major genotypes and random residuals. Under a classic polygenic model, this correlation is .5. Fit of the data under the mixed genetic model and restricted models is done using the computer program PAP: Pedigree Analysis Package (Hasstedt and Cartwright, 1981).

The fifth stage of analysis is a linkage investigation. The lod scores of spatial tests (if a major gene is indicated) with known gene markers are calculated using the computer program LIPED (Ott, 1974). The lod score method (Morton, 1955) involves calculating the likelihood of cosegregation of an unknown gene with a known gene at specified recombination values. The $\log$ of the ratio of likelihoods of specified recombination values, denoted by $\theta$, to that of independent assortment $(\theta=.5)$ is called a lod score and denoted by $z$.

\section{RESULTS}

\section{Cognitive Test Performance}

Univariate statistics for the six spatial tests are shown in Table II. Means and standard deviations of the spatial tests were similar to those 
Table II. Univariate Statistics of Six Spatial Tests ${ }^{a}$

\begin{tabular}{lcrrrrrr}
\hline Test & $N$ & $X$ & SD & $p$ & S & K & $r$ \\
\hline CR & 58 & 87.8 & 36.0 & 1.8 & 0 & .2 & .90 \\
CC & 58 & 15.1 & 10.9 & 1.0 & -.4 & .6 & .70 \\
MR & 65 & 13.1 & 8.2 & 1.0 & .5 & .8 & .67 \\
RF & 73 & 33.7 & 38.5 & $1.0^{b}$ & .3 & .1 & -.83 \\
GEF & 64 & 10.0 & 5.3 & 1.0 & -.3 & -1. & .93 \\
HP & 54 & 172.8 & 54.9 & 1.7 & 0 & -.2 & .90 \\
\hline
\end{tabular}

${ }^{a} \mathrm{p}$, power function in transformation of age/sex-adjusted scores; $S$, skewness; $\mathrm{K}$, kurtosis (deviation from normality); $r$, Pearson correlation coefficient of parts 1 and 2 of the test.

${ }^{b}$ Natural logarithm of raw RFT scores used; no subsequent power transformation after age/ sex adjustment required.

found in populations of unrelated subjects (Ekstrom et al., 1976; Vandenberg and Kuse, 1978; Witkin et al., 1971; Oltman, 1968), indicating that substantial phenotypic variation is present in the kindred. Sample sizes vary due to the inability of some subjects to attend both hours of testing $(N=7)$, age requirements, and mismarked test sheets $(N=1$ for GEF, $N=4$ for $\mathrm{HP}$ ).

\section{Pedigree/Segregation Analysis}

Variance component estimates under the general model $\left(V_{\mathrm{A}}, V_{\mathrm{D}}\right.$, and $V_{\mathrm{E}}$ present) are shown in Table III. Significant genetic variance is indicated for two spatial tests, RF and CR, using the likelihood-ratio criterion (Edwards, 1972). Furthermore, significant dominance variance is indicated for CR but not for any other spatial test. There is no genetic variance for MR or HP in this kindred, while additive genetic variance

Table III. Components of Variance on Six Spatial Tests ${ }^{a}$

\begin{tabular}{llccc}
\hline Test & \multicolumn{1}{c}{$V_{\mathrm{A}}$} & $V_{\mathrm{D}}$ & $V_{\mathrm{E}}$ & $\begin{array}{c}\chi^{2}(2 \mathrm{df}) \\
\left(V_{\mathrm{A}}=V_{\mathrm{D}}=0\right)\end{array}$ \\
\hline CC & $.68(.23)^{*}$ & $.00(.16)$ & $.41(.15)$ & 2.72 \\
CR & $.28(.20)^{*}$ & $.70(.18)^{* *}$ & $.01(.02)$ & $6.46^{* *}$ \\
GEF & $.36(.25)^{*}$ & $.00(.23)$ & $.63(.22)$ & 3.38 \\
HP & $.00(.12)$ & $.00(.17)$ & $.97(.19)$ & $<.01$ \\
MR & $.08(.14)$ & $.00(.16)$ & $.91(.17)$ & 0.18 \\
RF & $.55(.19)^{* * *}$ & $.00(.13)$ & $.45(.13)$ & $6.86^{* *}$ \\
\hline
\end{tabular}

${ }^{a} V_{\mathrm{A}}$, additive genetic variance (SE); $V_{\mathrm{D}}$, nonadditive genetic variance (SE); $V_{\mathrm{E}}$, nonfamilial environmental variance (SE).

$* p<.1$.

$* * p<.05$. 
Table IV. Segregation Analysis of CR and RF Under the Mixed Genetic Model ${ }^{a}$

\begin{tabular}{lccccccc}
\hline \multicolumn{1}{c}{ Model } & $q$ & $X_{\text {aa }}$ & $X_{\text {Aa }}$ & $X_{\text {AA }}$ & $h$ & $\sigma$ & $\chi^{2}(3 \mathrm{df})$ \\
\hline Card rotations & & & & & & & \\
MGM & .55 & -1.69 & .73 & -.05 & .47 & .65 & \\
PG & $(.11)$ & $(.26)$ & $(.22)$ & $(.21)$ & $(.31)$ & $(.08)$ & \\
& & -.18 & & & .31 & 1.01 & $14.28^{*}$ \\
Rod and frame & & $(.23)$ & & & $(.28)$ & $(.10)$ & \\
$\quad$ MGM & .37 & .31 & -.46 & 1.04 & .81 & .76 & \\
PG & $(.16)$ & $(.51)$ & $(.22)$ & $(.34)$ & $(.31)$ & $(.12)$ & \\
& & .21 & & & .58 & 1.02 & 2.39 \\
& & $(.21)$ & & & $(.22)$ & $(.10)$ & \\
\hline
\end{tabular}

${ }^{a}$ MGM-mixed genetic model; PG-polygenic model (no major gene: $X_{\mathrm{AA}}=X_{\mathrm{Aa}}=X_{\mathrm{aa}}$; $q=1.0) ; q$-frequency of allele $a ; X_{\text {aa }}, X_{\mathrm{Aa}}, X_{\mathrm{AA}}$-genotype means; $h$-heritability (i.e., polygenic variance/polygenic + environmental variance); $\sigma-$ common standard deviation of genotypes $A A, A a$, and $a a$.

$* p<.05$.

is significant for $\mathrm{RF}(p<.05)$ and marginally significant for $\mathrm{CC}(p<.1)$, and GEF $(p<.1)$.

Results from segregation analysis under the mixed genetic model for $\mathrm{CR}$ and RF are shown in Table IV. A major gene is indicated for CR from model comparisons of the mixed genetic model (MGM) and the restricted polygenic (PG) model. There is no evidence for presence of a major gene for RF based on model comparisons of MGM and PG.

We were unable to obtain maximum-likelihood estimates of the three transmission probabilities simultaneously for CR to evaluate the appropriateness of Mendelian values (i.e., the major gene model). However, we did calculate the maximum likelihood of the data under alternative models with one transmission probability fixed $\left(T_{1}, T_{2}\right.$, or $\left.T_{3}\right)$. Varying which transmission probability was fixed had little effect on the magnitude of the likelihood. We compared the fit of the kindred data on CR performance under one of these models (i.e., $T_{1}$ and $T_{2}$ free, $T_{3}$ fixed at zero) with the Mendelian model, and the chi-square statistic was not significant at the .05 level $\left(\chi_{2}^{2}=2.92\right)$.

However, the $95 \%$ confidence interval for $T_{2}$ under the less restricted model did not include the Mendelian value of .5 even though comparison with the Mendelian model was not significant. One explanation for this paradoxical finding is that ad hoc tests of single parameters (in this case, the equivalent of a Wald test) may not be exact when multiple parameters are simultaneously estimated and the sample size is small (Kendall and Stuart, 1960; Phillips, 1986). 
Table V. The lod Scores for Card Rotations and Autosomal Markers

\begin{tabular}{|c|c|c|c|c|c|c|c|c|c|}
\hline \multirow[b]{2}{*}{ Marker } & \multicolumn{6}{|c|}{$\theta$} & \multirow[b]{2}{*}{$Z_{\max }$} & \multirow[b]{2}{*}{$\theta_{\mathrm{m}}$} & \multirow[b]{2}{*}{$\theta_{\mathrm{f}}$} \\
\hline & .01 & .05 & .10 & .20 & .30 & .40 & & & \\
\hline$A B O$ & -1.53 & -.98 & -.72 & -.44 & -.26 & -.12 & & & \\
\hline$A C P$ & $.15^{a}$ & .15 & .14 & .11 & .08 & .04 & & & \\
\hline$A D A$ & .06 & .05 & .04 & .02 & .01 & .00 & & & \\
\hline$B F$ & -1.59 & -.97 & -.67 & -.36 & -.18 & -.07 & & & \\
\hline$E S D$ & -.16 & -.14 & -.11 & -.06 & -.03 & -.01 & & & \\
\hline$F Y$ & .02 & .01 & .01 & .01 & .00 & .00 & .04 & .01 & .50 \\
\hline GALT & -1.58 & -.98 & -.69 & -.39 & -.22 & -.10 & & & \\
\hline$G C$ & .44 & .42 & .39 & .32 & .23 & .13 & .51 & .50 & .01 \\
\hline$G P T$ & .25 & .26 & .26 & .24 & .19 & .11 & .44 & .50 & .01 \\
\hline$H P$ & -.13 & -.10 & -.07 & -.03 & -.01 & .00 & & & \\
\hline$I G H G(G M)$ & -.28 & -.25 & -.21 & -.14 & -.09 & -.04 & & & \\
\hline$J K$ & -1.57 & -.96 & -.67 & -.39 & -.22 & -.10 & & & \\
\hline$L E$ & $.01^{a}$ & .01 & .01 & .00 & .00 & .00 & & & \\
\hline$M N S$ & $.53^{a}$ & .48 & .42 & .29 & .17 & .07 & & & \\
\hline$P 1$ & -.35 & -.31 & -.26 & -.18 & -.10 & -.04 & & & \\
\hline$P G M 1$ & $.38^{a}$ & .32 & .26 & .17 & .10 & .04 & & & \\
\hline$P G P$ & -.16 & -.15 & -.13 & -.09 & -.06 & -.03 & & & \\
\hline PTC & -.09 & -.08 & -.07 & -.04 & -.03 & -.01 & & & \\
\hline$R H$ & $.03^{a}$ & .03 & .02 & .01 & .00 & .00 & & & \\
\hline
\end{tabular}

${ }^{a} Z_{\max }$ if it occurs at $\theta_{\mathrm{m}}=\theta_{\mathrm{f}}$; otherwise, $Z_{\mathrm{max}}, \theta_{\mathrm{m}}$, and $\theta_{\mathrm{f}}$ are given. Markers not shown had lod scores of zero at all $\theta$ values.

\section{Linkage Analysis}

We calculated lod scores for CR with the autosomal genetic markers and the $\mathrm{X}$-linked marker, $X G$, because results from the segregation analysis suggested a possible major gene. In addition, we calculated lod scores for GEF and RF with $X G$ because previous research suggested possible linkage of these spatial tests with $X G$ (Goodenough et al., 1977). Genotypic means and standard deviations for CR estimated under the codominant major gene model were used for calculating the lod scores. As shown in Table V, linkage of CR with 19 autosomal markers was relatively uninformative. The largest lod scores were found for $M N S$ and the $G C$ loci. $\operatorname{Lod}$ scores close to the rejection value of -2.0 were found for four loci, $A B O, J K, G A L T$, and $B F$, at male and female recombination values equal to .01 .

The lod scores for CR, RF, and GEF with $X G$ are shown in Table VI. We used genotypic means estimated under the autosomal codominant model as parameter estimates of female means in the X-linked model. The rationale for this is that genotypic ratios are equivalent for females under the two modes of transmission. Female homozygous means were 
Table VI. The lod Scores for $X G$ with CR, RF, and GEF ${ }^{a}$

\begin{tabular}{lrrrrrr}
\hline & \multicolumn{7}{c}{$\theta_{\mathrm{m}}=\theta_{\mathrm{f}}=$} \\
\cline { 2 - 7 } Trait & .01 & .05 & .10 & .20 & .30 & .40 \\
\hline CR & -.61 & -.51 & -.41 & -.27 & -.16 & -.07 \\
GEF & .11 & .10 & .08 & .06 & .04 & .02 \\
\hline
\end{tabular}

${ }^{a}$ RF completely uninformative in this family (i.e., lod scores all 0 ).

used as male genotypic mean estimates in LIPED. The family was completely uninformative for $X G$ and RF and relatively uninformative for the other two spatial tests.

\section{DISCUSSION}

Genetic analysis of six spatial tests in four generations of a single kindred offers little support for major gene involvement in spatial ability. Only one of six spatial tests, Card Rotations, had significant nonadditive genetic variance underlying task performance in the family. Segregation analysis of this test under a mixed genetic model suggested a major autosomal gene from model comparisons but this interpretation is equivocal based on the transmission probability estimates and their standard errors. Furthermore, we found the heterozygous mean to be greater than either homozygous mean. This apparent overdominance, we feel, may reflect a genotype-age interaction. Because of the small size of our sample, we could not test for a genotype-age interaction (Moll et al., 1984) and had to adjust for age assuming no interaction.

In contrast to findings supporting a major gene for Mental Rotations and Hidden Patterns in nuclear families (Ashton et al., 1979), genetic variance for these tests is negligible in this kindred. One explanation for this discrepancy is that a major gene for HP and MR is by chance not segregating in this family. An alternative explanation is that variance among sibships (due to shared environment and/or heterogeneity) in nuclear families leads to an overestimate of nonadditive gene effects and subsequent assignment to a major gene. A less plausible explanation is that a major gene is segregating for spatial ability but it has variable expression in families (MR and HP versus CR tasks).

We should further note that the power of our analyses is unknown. Although a single large extended kindred has advantages over small nuclear families in detecting a major gene through segregation patterns and linkage (discussed in the Introduction), there is no information on the 
specific power of such analyses in a pedigree of this size and structure. Simulation studies suggest that relatively large samples are required, total $N$ 's ranging from 450 to 675 , to obtain sufficient power in using the mixed model to detect a major gene (Burns et al., 1984). However, these simulation studies are based on a major gene with a relatively rare gene frequency (.012) compared to that expected for a trait such as spatial ability and on pedigrees ranging in size from 5 to 45 . However, given our sample size of 73 , insufficient power may have also contributed to these findings.

Linkage of Card Rotations with autosomal blood markers was inconclusive. The largest lod scores were found for two markers located on chromosome 4, $G C$ and $M N S$, but they were only marginally different from zero. Further investigations of cosegregation of spatial tests with these markers in other families may elucidate whether a major gene is present and located on chromosome 4 . The family was relatively uninformative for $X G$, thus precluding any conclusions regarding the proposed linkage (Goodenough et al., 1977) of RF and GEF with this marker.

\section{ACKNOWLEDGMENTS}

This research was in partial fulfillment of the requirements for the doctoral degree at the University of California (S.L.S.). We thank Dr. Sandra J. Hasstedt for her assistance with PAP, Drs. Katherine Neiswanger and Vivian Clemins for their invaluable aid in data collection, Nick Marinacci for drawing blood, and Drs. Mary Marazita and Art Woodward for many helpful suggestions. We are especially indebted to the wonderful family members who participated in this research.

\section{REFERENCES}

Ashton, G. C., Polovina, J. J., and Vandenberg, S. G. (1979). Segregation analysis of family data for 15 tests of cognitive ability. Behav. Genet 9:329-347.

Biomedical Computer Programs P-Series (1979). University of California Press, Los Angeles.

Burns, T. L., Moll, P. P., and Schork, M. A. (1984). Comparison of different sampling designs for the determination of genetic transmission mechanisms in quantitative traits. Am. J. Hum. Genet. 36:1060-1074.

Corah, N. L. (1965). Differentiation in children and their parents. J. Personal. 33:300-308.

DeFries, J. C., Johnson, R. C., Kuse, A. R., MeClearn, G. E., Polovina, J., Vandenberg, S. G., and Wilson, J. R. (1979). Familial resemblance for specific cognitive abilities. Behav. Genet. 9:23-43.

Eaves, L. J. (1983). Errors of inference in the detection of major gene effects on psychological test scores. Am. J. Hum. Genet. 35:1179-1189.

Edwards, A. W. F. (1972). Likelihood, Cambridge University Press, Cambridge.

Ekstrom, R. B., French, J. W., Harman, H. H., and Derman, D. (1976). Manual for Kit of Factor-Referenced Cognitive Tests, Education Testing Service, N.J.

Elston, R. C., and Bonney, G. E. (1984). Sampling considerations in the design and analysis of family studies. In Rao, D. C., Elston, R. C., Kuller, L. H., Feinlieb, M., Carter, 
C., and Havlik, R. (eds.), Genetic Epidemiology of Coronary Heart Disease: Past, Present, and Future, Alan R. Liss, New York, pp. 349-371.

Elston, R. C., and Stewart, J. (1971). A general model for the genetic analysis of pedigree data. Hum. Hered. 21:523-542.

Goodenough, D. R., Gandini, E., Olkin, I., Pizzamiglio, L., Thayer, D., and Witkin, H. A. (1977). A study of X chromosome linkage with field dependence and spatial visualization. Behav. Genet. 7:373-387.

Harris, H., and Kalmus, H. (1949). The measurement of taste sensitivity to phenylthiourea (PTC) Ann. Eugen. 15:24-31.

Hasstedt, S. J., and Cartwright, P. E. (1981). PAP: Pedigree Analysis Package, Revision 2, Technical Report No. 13, Department of Medical Biophysics and Computing, University of Utah, Salt Lake City.

Holt, S. B. (1968). The Genetics of Dermal Ridges, Charles C Thomas, Springfield, Ill.

Jardine, A. R., and Martin, N. G. (1984). No evidence of sex-linked or sex-limited gene expression influencing spatial orientation. Behav. Genet. 14:345-354.

Kendall, M., and Stuart, A. (1960). The Advanced Theory of Statistics: Inference and Relationship, Charles Griffin, London.

Lange, K. L., Westlake, J., and Spence, M. A. (1976). Extensions to pedigree analysis. III. Variance components by the scoring method. Ann. Hum. Genet. 39:485-491.

Loehlin, J. C., Sharon, S., and Jacoby, R. (1978). In pursuit of the "spatial gene": A family study. Behav. Genet. 8:27-41.

Moll, P. P., Sing, C. F., Lussier-Cacan, S., and Davignon, J. (1984). An application of a model for a genotype-dependent relationship between a concomitant (age) and a quantitative trait (LDL cholesterol) in pedigree data. Genet. Epid. 1:301-314.

Morton, N. E. (1955). Sequential tests for the detection of linkage. Am. J. Hum. Genet. $7: 277-318$.

Morton, N. E., and MacLean, C. J. (1974). Analysis of family resemblance in complex segregation of quantitative traits. Am. J. Hum. Genet. 26:489-503.

O'Connor, J. (1943). Structural Visualization, Human Engineering Lab, Boston.

Oltman, P. K. (1968). A portable rod and frame apparatus. Percept. Motor Skills 26:503506.

Ott, J. (1974). Estimation of the recombination fraction in human pedigrees: Efficient computation of the likelihood for human linkage studies. Am. J. Hum. Genet. 26:588-597.

Phillips, P. C. B. (1986). The exact distribution of the Wald statistic. Econometrica 54:881895.

Shepard, R. N., and Metzler, J. (1971). Mental rotation of three-dimensional objects. Science 171:701-703.

Tukey, J. W. (1977). Exploratory Data Analysis, Addison-Wesley, Reading, Mass.

Vandenberg, S. G., and Kuse, A. R. (1978). Mental rotations, a group test of three-dimensional spatial visualization. Percept. Motor Skills 47:599-604.

Vandenberg, S. G., and Kuse, A. R. (1979). Spatial ability: A critical review of the sexlinked major gene hypothesis. In Wittig, M. A., and Petersen, A. C. (eds.), Sex Related Differences in Cognitive Functioning, Academic Press, New York, pp. 67-95.

Wilson, J. R., and Vandenberg, S. G. (1977). Sex differences in cognition: evidence from the Hawaii Family Study. In McGill, T. E., Dewsburg, D. A., and Sachs, B. D. (eds.), Sex and Behavior, Plenum, New York, pp. 317-335.

Witkin, H. A., Oltman, P. K., Raskin, E., and Karp, S. A. (1971). A Manual for the Embedded Figures Tests, Consulting Psychologists Press, Palo Alto, Calif.

Edited by N. G. Martin 